\title{
Increased Peripheral TFH Cells and Serum Interleukin-21 in Childhood Mycoplasma Pneumoniae Pneumonia
}

\author{
Haiyan Xing ${ }^{1}$, Xiaoyan $\mathrm{Liu}^{2}$, Aimin $\mathrm{Li}^{2}$, Chunxiang $\mathrm{Li}^{2}$ and Jianyong Wang ${ }^{2}$ \\ ${ }^{1}$ Department of Allergy, The Affiliated Yantai Yuhuangding Hospital of Qingdao University, China. \\ ${ }^{2}$ Department of Pediatrics, The Affiliated Yantai Yuhuangding Hospital of Qingdao University, China.
}

\begin{abstract}
The aim of this study was to investigate the role of TFH cells and interleukin (IL)-21 in the pathogenesis of childhood Mycoplasma pneumoniae pneumonia (MPP).

It was a retrospective study carried out at Yantai Yuhuangding Hospital, China from July 2016 to June 2019. It comprised 46 healthy control (HC) children and 106 pediatric patients, including 60 children with general MPP (GMPP) and 46 children with refractory MPP (RMPP). Flow cytometry was used to detect the frequencies of peripheral TFH cells. The levels of serum IL-21 were tested by ELISA. The transcriptional factors Bcl- 6 and Blimp-1 mRNA levels were examined using RT-qPCR. The percentage of TFH cells and expression levels of IL-21 and BCl-6 in peripheral blood were all significantly elevated in MPP groups compared with HC group (all $p<0.001$ ). Furthermore, it was found that a significantly higher percentage of TFH cells and levels of IL-21 and $\mathrm{BCl}-6$ in RMPP children, compared with GMPP children (all $\mathrm{p}<0.05$ ). In contrast, lower Blimp-1 levels were observed in MPP groups $(p<0.001)$, especially in RMPP group $(p=0.021)$. Therefore, the elevated expansion of TFH cells and the increased secretion of IL-21 cytokine may contribute to the pathogenesis of childhood MPP, while its excessive response may be associated with the development of childhood RMPP.
\end{abstract}

Key Words: TFH cells, IL-21, Children, Mycoplasma pneumoniae pneumonia.

How to cite this article: Xing H, Liu X, Li A, Li C, Wang J. Increased Peripheral TFH Cells and Serum Interleukin-21 in Childhood Mycoplasma Pneumoniae Pneumonia. J Coll Physicians Surg Pak 2021; 31(05):605-607.

Mycoplasma pneumoniae (MP) is a significant cause of respiratory tract infections in children, causing atypical pneumonia. Although MP pneumonia (MPP) is generally a benign self-limiting illness, sometimes it can develop into refractory MPP (RMPP), presenting prolonged fever coupled with the radiological deterioration despite proper treatment with macrolide; and can result in severe, life-threatening pneumonia. ${ }^{1}$ Although the potential mechanisms of MPP are still unclear, previous studies have demonstrated that the excessive immunological inflammation, including the overexpression of cytokines and highly disordered CD4+ T helper (Th) cells mediated immune reactions, may play a very vital role in the pathogenic mechanisms. ${ }^{2}$ Follicular Th (TFH) cells, a distinct subset of CD4+ Th cells that regulate humoral immune response, are located in $\mathrm{B}$ cell follicles of the secondary lymphoid organs. ${ }^{3}$ TFH cells are specialised providers of $\mathrm{T}$ cells, help to $\mathrm{B}$ cells and play prominent roles in the development of germinal center (GC), memory B cells and most high-affinity antibodies.

Correspondence to: Dr. Jianyong Wang, Department of Pediatrics, The Affiliated Yantai Yuhuangding Hospital of Qingdao University, China.

E-mail: ekwjy2012@163.com

Received: April 02, 2020; Revised: October 02, 2020;

Accepted: December 28, 2020

DOI: https://doi.org/10.29271/jcpsp.2021.05.605
Importantly, TFH cells are recognised to critically contribute to protective immunity against pathogens and autoimmune diseases. ${ }^{3}$ However, no study has investigated the roles of TFH cells in the pathogenesis of childhood MPP.

In this research, the aims were to evaluate the roles of TFH cells in the pathogenesis of children with MPP through examining the percentage of TFH cells and expression levels of IL-21 and related transcript factors Bcl-6 and Blimp-1 in general MPP (GMPP) patients and RMPP patients.

This retrospective study was conducted at Yantai Yuhuangding Hospital, China, from July 2016 to June 2019 , and was approved by the Hospital Ethics Committee; and written informed consent was acquired from guardians of all children. A total of 106 children with MPP were enrolled. Diagnosis of pneumonia was based on clinical features and radiological findings. Infection with MP was confirmed by an initial MP-IgM titer, no less than 1:160 and/or a positive PCR result. RMPP was diagnosed as fever lasting for 7 days or more, with clinical as well as radiological deterioration despite appropriate macrolide therapy. ${ }^{1}$

The exclusion criteria for the study were: patients co-infected with other pathogens; patients who accepted hormones before collecting samples; and children who had underlying diseases (chronic pulmonary disease, asthma, recurrent respiratory tract infection, cardiovascular disease, rheumatic diseases, tumor, congenital disease, and immunodeficiency). 
Table I: Clinical characteristics of the MPP children in this study.

\begin{tabular}{|c|c|c|c|c|}
\hline Variable & HC $(n=46)$ & GMPP $(n=60)$ & RMPP $(n=46)$ & p-value \\
\hline \multicolumn{5}{|l|}{ Demographic } \\
\hline Age (months) & $82.38 \pm 28.71$ & $78.85 \pm 30.29$ & $85.57 \pm 33.56$ & 0.354 \\
\hline Gender (female/male) & $22(47.8 \%) / 24(52.2 \%)$ & $33(55 \%) / 27(45 \%)$ & $21(45.7 \%) / 25$ (54.3\%) & 0.596 \\
\hline \multicolumn{5}{|l|}{ Symptoms } \\
\hline Fever duration (days) & & $7.79 \pm 4.22$ & $11.95 \pm 5.36$ & $<0.001$ \\
\hline Tachypnea & & $2(3.3 \%)$ & $13(28.3 \%)$ & $<0.001$ \\
\hline Extrapulmonary complications & & $7(11.7 \%)$ & $14(30.4 \%)$ & 0.016 \\
\hline \multicolumn{5}{|l|}{ Laboratory findings } \\
\hline WBC $(\times 109 / L)$ & & $8.87 \pm 3.89$ & $10.41 \pm 6.34$ & 0.085 \\
\hline CRP (mg/L) & & $7.83 \pm 6.86$ & $39.08 \pm 35.15$ & 0.006 \\
\hline High level of LDH ( $\geq 410$ IU/L) & & $8(13.3 \%)$ & $18(39.1 \%)$ & 0.002 \\
\hline \multicolumn{5}{|l|}{ Radiology } \\
\hline Lobar pneumonia & & $21(35.0 \%)$ & $36(78.3 \%)$ & $<0.001$ \\
\hline Pleural effusion & & $10(16.7 \%)$ & $22(47.8 \%)$ & 0.001 \\
\hline
\end{tabular}

Table II: Comparison of the levels of TFH cells, IL-21, relative Bcl-6 and Blimp-1 mRNA in each group.

Table II: Comparison of the levels of TFH cells, IL-21, relative Bcl-6 and Blimp-1 mRNA in each group.
\begin{tabular}{|l|c|c|c|c|c|}
\hline Variable & HC & GMPP & RMPP & p-value* & p-value** \\
\hline TFH cells (\%) & $7.95 \pm 2.39$ & $14.07 \pm 4.61$ & $18.56 \pm 4.22$ & $<0.001$ & $<0.001$ \\
\hline IL-21 (pg/ml) & $102.95 \pm 30.02$ & $146.52 \pm 35.63$ & $178.73 \pm 37.54$ & $<0.001$ & $<0.001$ \\
\hline Bcl-6 mRNA & $0.97 \pm 0.45$ & $1.41 \pm 0.67$ & $1.77 \pm 0.82$ & $<0.001$ & 0.045 \\
\hline Blimp-1 mRNA & $1.03 \pm 0.32$ & $0.83 \pm 0.39$ & $0.66 \pm 0.35$ & $<0.001$ & 0.021 \\
\hline${ }^{*} p$-value for the three groups (HC, GMPP and RMPP); ${ }^{* *} p$-value for comparison between GMPP and RMPP groups. \\
\hline
\end{tabular}

Forty-six healthy children who underwent routine physical examination during the same period were selected as healthy control $(\mathrm{HC})$ group.

Blood samples of children with MPP were obtained before treatment. Venous blood samples were gathered on an empty stomach from individual HC and MPP children. Then, peripheral blood mononuclear cells (PBMCs) were purified using density gradient centrifugation with Ficoll-paque plus (GE healthcare, Sweden), and serum samples were separated and stored at $-80^{\circ} \mathrm{C}$ for detection of ELISA.

Human PBMCs were immune stained with PE-anti-CD4 and FITC-anti-CXCR5 (eBioscience, USA) at room temperature for 30 mins. Isotype antibodies were given as controls. The cells were washed with PBS and analysed by flow cytometry (FACSAria II, Becton Dickinson). TFH cells were identified as those that were CD4+ CXCR5 +. The concentrations of serum IL-21 were measured by ELISA (eBioscience, USA).

Total RNA of PBMCs was prepared using trizol reagent (Invitrogen, USA). Reverse transcription kit was used to synthesise complementary DNA. PCR amplifications were performed in triplicate using primer and SYBR Green Master Mix on LightCycler ${ }^{\circledR} 480$ (all from Roche). The primers used for the RT-qPCR were: BCl-6: sense, 5'-ACC AGA ATG CCA AAC CAG AG-3', and antisense, 5'-TCA AGG TTC TCA GGC TCC AT-3'; Blimp-1: sense, 5'-AGC AGC GAC GAA GCC ATG AAT C-3' and antisense, 5'-TGC ACT CTC AGG TGG ACC TTC AG-3'. The relative mRNA expression was calculated using the the 2- $\Delta \Delta \mathrm{Ct}$ method, and the results were normalised using GAPDH as the reference gene.

Data were analysed using SPSS software (version 20.0).
The Shapiro-Wilk test was used to assess the normality of quantitative data. Continuous variables with a normal distribution were presented as mean $\pm \mathrm{SD}$, and an overall variation among the different groups was analysed by one-way ANOVA; and student's t-test was chosen between two groups. Categorical data were expressed as frequencies and percentages, and Chi-square / Fisher's Exact test was used to compare categorical data. Statistical significance was defined as $p<0.05$.

A total of 152 participants were included in this research, and their clinical characteristics were described in Table I.

As shown in Table II, the TFH cells percentage (CD4+ CXCR5 +/CD4+ T cells) in PBMCs displayed significantly increasing in MPP groups ( $p<0.001$ ), with significant differences between GMPP and RMPP groups ( $p<0.001$ ). The levels of serum IL-21 were also significantly enhancive in MPP groups ( $p<0.001$ ), and were higher in RMPP group than in GMPP group ( $p<0.001$ ). The relative mRNA levels of Bcl-6 and Blimp-1 were compared among the three groups. The RMPP group performed highest relative $\mathrm{Bcl}-6$ mRNA levels among the three groups, followed by GMPP group ( $p$ $<0.05$ ), while the lowest values were occurred in $\mathrm{HC}$ group $(p<0.001)$. On the contrary, the relative mRNA levels of Blimp-1 showed significantly decreasing trends in HC, GMPP and RMPP groups in order (both $p<0.05$ ).

Previous studies have revealed that the developments of MPP, particularly the RMPP, are closely associated with the excessively activated cell-mediated immune response. For example, the imbalance of peripheral blood Th17 and Treg cells is closely related to the deterioration of pulmonary 
injury in childhood MPP. ${ }^{2}$ Here, this study provided evidences for the first time that TFH cells might be involved in the progression of MPP, particularly the RMPP.

As a special subset of CD4+ Th cells, TFH cells are characterised by the expression of numerous molecules including CXCR5, cytokines of IL-21 and transcription factor $\mathrm{BCl}-6$. TFH cells play a key role in adjusting B-cell activation, germinal center formation, and heightening humoral immunity. Owing to the essential contribution by TFH cells to the humoral immunity, they have an obviously important role in defense against pathogens. Elevated TFH cells have been shown to play protective roles in different viral and parasite infections. ${ }^{4,5}$ In this research, the elevated frequencies of TFH cells in MPP children compared with healthy children suggested the involvement of TFH cells in the defensing MP infection. Additionally, high-level IL-21 excretion is a distinctive characteristic of TFH cells, and IL-21 is pivotal for the development of TFH cells. Moreover, Bcl-6 is the "master" transcription factors for the multistage, multifactorial TFH cells differentiation. ${ }^{3}$ In contrast, the transcription factor Blimp-1, an antagonist of $\mathrm{BCl}-6$, inhibits TFH cells differentiation. ${ }^{3}$ Therefore, the increased expression levels of $\mathrm{Bcl}-6$ and IL-21 in PBMCs in MPP children compared with healthy children further supported the involvement of TFH cells in defensing MP infection, which was also supported by the decreased levels of Blimp-1 in PBMCs in MPP children.

On the other hand, the elevated expansion of TFH cells and the increased secretion of IL-21 not only boost immune responses, but also induce autoimmune disturbance. Increased frequencies of circulating TFH cells and concentrations of serum IL-21 were observed in subsets of patients with asthma, immune thrombocytopenia, Henoch-Schonlein purpura nephritis, Sjogren's syndrome, rheumatoid arthritis, systemic lupus erythematosus, juvenile dermatomyositis, autoimmune thyroid disease, and inflammatory bowel disease. ${ }^{6}$ Those studies have demonstrated the overexpression of TFH cells and IL-21 play an important and complicated role in the initiation and progression of inflammatory and autoimmune diseases. Therefore, TFH cells and IL-21 levels in RMPP group were much higher than those in
GMPP group in this study, probably indicating that TFH cells might be involved in the development of RMPP, which was also agreed by the expression changed of transcript factors Bcl-6 and Blimp-1.

To sum up, TFH cells and IL-21 involved in the pathogenesis of childhood MPP, while its excessive response may be associated with the development of childhood RMPP. This study may supply more efforts for understanding the pathogenic mechanisms of MPP.

\section{CONFLICT OF INTEREST:}

The authors declared no conflict of interest. AUTHORS' CONTRIBUTION:

HX, XL: Data statistics and analysis, drafting the manuscript, revision, read and approved the final manuscript.

AL, CL: Data collection, revision, discussion, read and approved the final manuscript.

JW: Design, supervision, revision, and final approval.

\section{REFERENCES}

1. Wang M, Wang Y, Yan Y, Zhu C, Huang L, Shao X, et al. Clinical and laboratory profiles of refractory Mycoplasma pneumoniae pneumonia in children. Int J Infect dis 2014; 29:18-23. doi: 10.1016/j.ijid.2014.07.020.

2. Guo H, He Z, Li M, Wang T, Zhang L. Imbalance of peripheral blood Th17 and Treg responses in children with refractory Mycoplasma pneumoniae pneumonia. J Infect Chemother 2016; 22(3):162-6. doi: 10.1016/j.jiac.2015. 12.006.

3. Vinuesa CG, Linterman MA, Yu D, MacLennan IC. Follicular Helper T Cells. Annual Review Immunol 2016; 34:335-68. doi.org/10.1146/annurev-immunol-041015-055605.

4. Wan Z, Zhou Z, Liu Y, Lai Y, Luo Y, Peng X, et al. Regulatory $T$ cells and T helper 17 cells in viral infection. Scandinavian J Immunol 2020; 91(5):e12873. doi: 10.1111/sji.12873.

5. Kumar R, Loughland JR, Ng SS, Boyle MJ, Engwerda CR. The regulation of CD4 $(+)$ T cells during malaria. Immunological Rev 2020; 293(1):70-87. doi: 10.1111/imr.12804.

6. Gensous N, Charrier M, Duluc D, Contin-Bordes C, Truchetet ME, Lazaro $E$, et al. T follicular helper cells in autoimmune disorders. Front Immunol 2018; 9:1637. doi: 10.3389/ fimmu.2018.01637. 